\title{
Anthropogenic Climatic Change Risks a Global Anthrax Outbreak : A Short Communication
}

\section{Jia B Kangbai* and Edwin Momoh}

Center for International Health, Ludwig Maximilians University Munich, Bayern, Germany

*Corresponding author: Kangbai JB, Center for International Health, Ludwig Maximilians University Munich, Bayern, Germany, Tel: +49347856; Fax: +49347856; Email: Jia.Kangbai@Irz.uni-muenchen.de

Received date: July 28, 2017; Accepted date: August 06, 2017; Published date: August 18, 2017

Copyright: ( 2017 Kangbai JB, et al. This is an open-access article distributed under the terms of the Creative Commons Attribution License, which permits unrestricted use, distribution, and reproduction in any medium, provided the original author and source are credited.

\section{Introduction}

Global warming is predicted to be responsible for the unprecedented changes to the global climate. Temperate regions like North America and the Arctic regions are now experiencing warmer temperatures, more rainfall and droughts, as well as extreme weather including hurricanes and tornadoes [1]. These climate-related events will invariable change the incidence and distribution of emerging infectious diseases [2]. Emerging infectious disease is defined as any infection that appears in a population or that is rapidly increasing in incidence or geographic range [2]. Infections like hantavirus pulmonary syndrome, HIV/AIDS, Lyme disease, and hemolytic uremic syndrome are the mostly talked about emerging infectious diseases these days. However, disease like anthrax that is periodical or have taken a long time since its last outbreak could also be discribed as an emerging infection.

\section{Global Outbreak of Human Anthrax}

Anthrax is caused by Bacillus anthracis and is an anthropozoonotic infection. It is a highly virulent disease with both invasive and toxicogenic infection pathways in which toxins play a major role in its pathogenicity [3]. The infection is fatal in animals and humans when the Bacillus anthracis endospores enter the body either by inhalational, or through cutaneous openings, or by ingestion [4]. Because Bacillus anthracis endospores persist for a very long time in the environment, coupled with their ease of in vitro production, they can be easily designed in to biological weapons. Japan, USA, United Kingdom and Russia have had anthrax form of biological weapon [5]. Human anthrax used to kill thousands of people and livestock prior to Loiuis Pasteur's discovery of its vaccine in the $20^{\text {th }}$ century [6], but the disease is now posing new threat in the wake of global warming and climate change.

The world is still at risk for a global outbreak of human anthrax on the scale of the 2014 West African Ebola outbreak. Zimbabwe recorded the largest non-military anthrax epidemic in modern time (1979) with over 9,400 cases and 182 fatalities [7]. The most recent human anthrax outbreak occurred in Siberia in 2016 resulting to the deaths of over two thousand livestock mainly reindeers and one young boy. The Siberian anthrax outbreak was blamed on climate change which experts believe caused deadly anthrax endospores to reemerge following years been frozen in the permafrost [8].

Several factors including ecological, environmental and demographic profiles increases the risk for a global anthrax outbreak. Although anthropogenic factors is principal in precipitating an anthrax outbreak it alone is insufficient. Human factors can be confounded by climatic conditions. Climatic factors generally can act on nonanthropongenic factors to increase the prevalence of anthrax in some locale; this increase, alongside the spontaneous viral evolution as well as the emergence of microbial variants will compound an anthrax outbreak by increasing the stake of an anthrax antibiotic drug resistance strain.

Climate change can determine the habitat range, population size and ninche of livestock and human, the convergence of infected human host, transmission season and the timing and persistence of an anthrax outbreak. As temperature increases and rainfall patterns are altered, livestocks that serves as anthrax primary hosts will shift their habitat and come to live closer to humans hence promoting the geographic occurrence of the infection in a new terrain. Additionally, changes in climate leading to environmental crisis such as desertification and flooding which eventually leads to both human population migration and behavioural change might also indirectly affect the direct spread of the disease into some areas.

Also, agreed that person-to-person transmission of anthrax is extremely unlikely this could not be ruled out when there is an outbreak in the face of huge climatic and envirnmental disasters. Despite that, recent claims that cutaneous anthrax transmission can occur as a result of direct contact with infectious discharges from a cutaneous lesions of an anthrax patient has compounded the transmission pathways of anthrax.

Controlling and managing a global anthrax outbreak could be cumbersome primarily because of the difficulty in diagnosing the infection. The difficulties in designing an effective anthrax diagnostic techniques including molecular based diagnostic method is in part associated with the emergence of Bacillus cereus and Bacillus thuringiensis [9]. Bacillus anthracis is similar to both Bacillus cereus and Bacillus thuringiensis which are also pathogenic to humans. This similarity in pathogenecity makes it hard to assess and analyze the risk of a human anthrax outbreak since the infections are all zoonosis. Additionally, because there are often few cases of human anthrax cases in the previous outbreaks interest in developing rapid diagonistic techniques which can serves as an effective risk assessment tool appears lacking. This challenge in designing an effective, rapid diagonistic and risk assessment tool for human anthrax is both a public health and military concern considering the fact that human anthrax can be weaponized into a bio-weapon. And as man becomes more vulnerable to the effects of climate change these concerns are expected to increase.

Man's vulnerability to the potential health impact of a climate change related global anthrax outbreak may be effectively controlled by adopting measures which includes mitigating the effects of climate change, improve better understanding of climate change related anthrax-specific health problems, investing in innovative and effective climate change monitoring technologies, as well as engage in efficient 
Citation: Kangbai JB, Momoh E (2017) Anthropogenic Climatic Change Risks a Global Anthrax Outbreak : A Short Communication. J Trop Dis 5:

Page 2 of 2

resources allocation in order to promote healthy lifestyles and raise public awareness.

Because anthropogenic climatic change risk increasing the incidence of emerging infectious disease, by expanding the geographic ranges of pathogenic zoonotic species, evolution of pathogen dynamics in environmental, livestock and human reservoirs, it is prudent man desgin strategies to mitigate its impact. To tackle the potential health impact of a climate change-induced anthrax outbreak medical and public health communities should be prepared to handle such threats in the first place.

\section{Conclusion}

Firstly, public health communities and other health-related agencies must have the ability to be able to measure changes in emerging infectious disease burden. This is justified because several diseases such as Lyme disease, hantavirus pulmonary syndrome, and hemolytic uremic syndrome classically termed as emerging infectious diseases are mostly not subject to national disease surveillance making their reporting difficult. It is thus helpful to reconsider the status of several of these important diseases in relation to national disease surveillance.

At community level the wideranging and unpredictable health effects that results from an anthropogenic climatic change highlights the need for the strengthening of disease surveillance and public health outbreak response infrastructures. Medical practitioners, physicians and other health care providers generally recognised as credible opinion leaders and advocates on environmental issues can be made to enhance the health of the communities they are serving by engaging in awareness raising and sensitization campaigns.

\section{References}

1. US Environmental Protection Agency (2016) Climate change indicators: Weather and Climate.

2. Flahault A, Castaneda RR, Bollon I (2016) Climate change and infectious diseases. Public Health Reviews 37: 21.

3. Levy H, Glinert I, Weiss S, Sittner A, Schlomovitz J, et al. (2014) Toxinindependent virulence of Bacillus anthracis in rabbits. PLoS One 9: 84947.

4. Kalamas AG (2004) Anthrax. Anesthesiol Clin North America 22: 533-540.

5. Ramesh Gupta (2009) Handbook of Toxicologz of chemical warfarre agents.

6. Smith KA (2005) Wanted, an Anthrax vaccine: Dead or alive? Med Immunol 4: 5.

7. Davies JC (1982) A major epidemic of anthrax in Zimbabwe. Cent Afr J Med 28: 291-298.

8. The Daily Kos (2016) The deadly anthrax outbreak in Siberia due to climate change.

9. Ogawa H, Fujikura D, Ohnuma M, Ohnishi N, Hang'ombe BM, et al. (2015) A novel multiplex PCR discriminates Bacillus anthracis and its genetically related strains from other bacillus cereus group species. PLoS One 10: 0122004. 